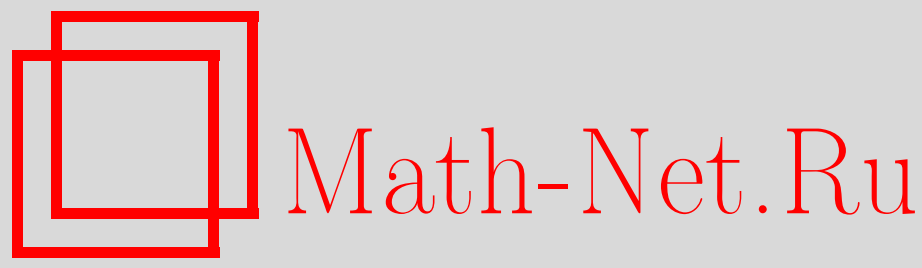

В. В. Борзов, Обобщенный осциллятор и его когерентные состояния, ТМФ, 2007, том 153, номер 3, 363-380

DOI: https://doi.org/10.4213/tmf6142

Использование Общероссийского математического портала Math-Net.Ru подразумевает, что вы прочитали и согласны с пользовательским соглашением http: //www.mathnet.ru/rus/agreement

Параметры загрузки:

IP: 54.162 .127 .20

26 апреля 2023 г., 15:54:28

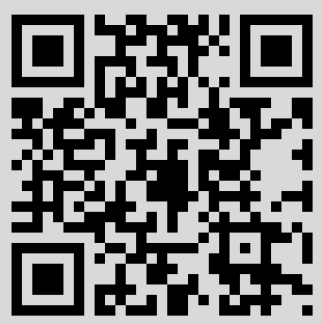




\section{ОБОБЩЕННЫЙ ОСЦИЛЛЯТОР И ЕГО КОГЕРЕНТНЫЕ СОСТОЯНИЯ}

Построена система, подобная осциллятору (обобщенный осциллятор), связанная с системой ортогональных полиномов на вещественной оси. Определены когерентные состояния в пространстве Фока соответствующего обобщенного осциллятора. На примере обобщенного осциллятора, связанного с полиномами Гегенбауэра, доказана (сверх)полнота этих когерентных состояний, т.е. построена мера, определяющая разложение единицы. Приведена формула, позволяющая вычислить параметр Манделя для построенных когерентных состояний.

Ключевые слова: ортогональные полиномы, гармонический осциллятор, обобщенный осциллятор, операторы рождения и уничтожения, когерентные состояния, параметр Манделя.

\section{1. ВВЕДЕНИЕ}

Круг вопросов, затронутых в работе, связывает между собой три основных понятия: ортогональные многочлены, простейшие системы квантовой механики такие, как обычный гармонический осциллятор, и, наконец, те состояния квантовой механики (наиболее близкие к классическим), которые называются когерентными. Ортогональные многочлены [1]-[3] используются практически во всех разделах современной математической и теоретической физики, в частности многочлены Эрмита, которые (после умножения на $e^{-x^{2}}$ ) образуют систему собственных функций оператора энергии гармонического осциллятора. Гармонический осциллятор и связанная с ним алгебра Гейзенберга являются фундаментальными понятиями квантовой теории. В последние десятилетия в квантовой физике (особенно в квантовой оптике) стали использоваться некоторые обобщения понятия гармонического осциллятора [4]-[9]. Имеются две основные возможности для такого обобщения. Первая использование более общего уравнения (дифференциального или разностного) в качестве уравнения Шредингера (см., например, работу [5]). Вторая - использование

${ }^{*}$ Санкт-Петербургский государственный университет телекоммуникаций, Санкт-Петербург, Россия. E-mail: Vadim@VB6384.spb.edu 
более общих (деформированных) перестановочных соотношений [6]-[9]. Заметим, что в обоих случаях ортогональные полиномы возникают при решении уравнения Шредингера или при изучении неприводимых представлений алгебры рассматриваемого осциллятора.

В настоящей работе рассматривается новый подход к изучению связи между ортогональными полиномами и алгебрами некоторых осцилляторов [10]. А именно, мы покажем, что многочлены Эрмита не являются исключением, так как каждой системе ортогональных многочленов на вещественной оси можно сопоставить систему, подобную осциллятору [10]. При этом мы определяем соответствующий осциллятор и его гамильтониан по заданному набору ортогональных многочленов, которые оказываются собственными функциями гамильтониана, в то время как при стандартном подходе сам осциллятор и его оператор энергии известны, а следует искать систему собственных функций. Заметим, что понятие "обобщенного" осциллятора возникает как естественное обобщение широко используемого в современной литературе понятия "деформированного" осциллятора [7]-[9] и сохраняет основные черты алгебры “деформированного" осциллятора. Однако в отличие от "деформированных" осцилляторов, где при предельном переходе параметров (к 0 или к 1) “деформированная" алгебра переходит в алгебру Гейзенберга, в нашем случае отсутствует связь с алгеброй обычного гармонического осциллятора. Тем не менее для некоторых систем ортогональных полиномов, зависящих от параметра, предельный переход по параметру можно трактовать как снятие деформации. Ниже мы остановимся на основных аналогиях, которые дают право использовать термин “обобщенный” осциллятор.

Рассматривается гильбертово пространство функций, квадратично-суммируемых на вещественной оси, в котором исследуемая система полиномов образует ортонормированный базис. Трехчленные рекуррентные соотношения для этих полиномов определяют матрицу Якоби для оператора “координаты” $X$. С помощью ядра Пуассона для данной системы полиномов определяется обобщенное преобразование Фурье, которое позволяет ввести оператор "импульса" $P$, причем справедлива обычная связь между операторами "координаты" $X$ и "импульса" $P$. Более того, это преобразование оставляет инвариантным оператор энергии (гамильтониан) $H=X^{2}+P^{2}$. Оператор $H$ имеет простой дискретный спектр, причем собственными функциями этого оператора являются исходные ортогональные полиномы. Собственные значения оператора $H$ (уровни энергии) вычисляются для всех известных систем ортогональных полиномов. За исключением гармонического осциллятора уровни энергии не эквидистантны.

Будем рассматривать заданное гильбертово пространство как реализацию пространства Фока. Лестничные операторы (рождения и уничтожения) $a^{+}$и $a^{-}$строятся обычным образом из самосопряженных операторов "координаты" $X$ и "импульса" $P$. Кроме них определяется оператор числа $N$ в этом пространстве Фока. Эти 
операторы удовлетворяют перестановочным соотношениям, которые являются обобщением соотношений Гейзенберга, и служат генераторами некоторой алгебры, которую естественно называть алгеброй “обобщенного” осциллятора.

\section{2. СИММЕТРИЧНАЯ СХЕМА}

Рассматривается положительная борелевская мера $\mu$ на вещественной оси $\mathbb{R}^{1}$, для которой конечны все моменты

$$
\mu_{n}=\int_{-\infty}^{\infty} x^{n} \mu(d x), \quad n=0,1, \ldots,
$$

причем справедливы соотношения

$$
\mu_{0}=1, \quad \mu_{2 k+1}=0, \quad k=0,1, \ldots
$$

Будем называть такую меру $\mu$ симметричной вероятностной мерой и обозначим как $\mathcal{H}_{\mu}=L^{2}\left(\mathbb{R}^{1} ; \mu(d x)\right)$ гильбертово пространство квадратично-суммируемых функций по мере $\mu$. По заданной последовательности моментов $\left\{\mu_{2 n}\right\}_{n=0}^{\infty}$ единственным образом определяется положительная последовательность $\left\{b_{n}\right\}_{n=0}^{\infty}, b_{n}>0, n=0,1, \ldots$, как решение некоторой алгебраической системы уравнений [10].

ОПреДЕЛЕНИЕ 2.1. Система вещественных полиномов $\left\{\psi_{n}(x)\right\}_{n=0}^{\infty}$ называется "канонической” относительно симметричной вероятностной меры $\mu$, если она определяется по положительной последовательности $\left\{b_{n}\right\}_{n=0}^{\infty}$ с помощью следующих рекуррентных соотношений:

$$
\begin{gathered}
x \psi_{n}(x)=b_{n} \psi_{n+1}(x)+b_{n-1} \psi_{n-1}(x), \quad n \geqslant 0, \quad b_{-1}=0, \\
\psi_{0}(x)=1 .
\end{gathered}
$$

Соотношения (2.3) определяют симметричную матрицу Якоби

$$
J=\left\{b_{i j}\right\}_{i, j=0}^{\infty}
$$

у которой отличны от нуля только элементы $b_{i, i+1}=b_{i+1, i}=b_{i}>0, i=0,1, \ldots$.

Имеет место следующая теорема.

Теорема 2.1. Пусть $\mu$-симметричная вероятностная мера на $\mathbb{R}^{1}$, и система вещественных полиномов

$$
\left\{\psi_{n}(x)\right\}_{n=0}^{\infty}
$$

- каноническая относительно меры $\mu$. Тогда система (2.5):

1) ортонормирована относительно меры $\mu$;

2) полна в гилъбертовом пространстве $\mathcal{H}_{\mu}=L^{2}\left(\mathbb{R}^{1} ; \mu(d x)\right)$ только в том случае, когда мера $\mu$ является $N$ - экстремальным решением проблемы моментов, порождаемой матрицей $J$. 
Первая часть утверждения теоремы проверяется непосредственными вычислениями, вторая эквивалентна теореме 2.3.3 из монографии [11].

Обозначим через $\mathcal{H}_{\mu}^{(1)}$ и $\mathcal{H}_{\mu}^{(2)}$ две копии пространства $\mathcal{H}_{\mu}$ :

$$
\mathcal{H}_{\mu}^{(1)}=L^{2}\left(\mathbb{R}^{1} ; \mu(d x)\right), \quad \mathcal{H}_{\mu}^{(2)}=L^{2}\left(\mathbb{R}^{1} ; \mu(d y)\right) .
$$

Ядро Пуассона $\mathfrak{K}_{\mathcal{H}_{\mu}}(x, y ; t)$ определяется формулой

$$
\mathfrak{K}_{\mathcal{H}_{\mu}}(x, y ; t)=\sum_{n=0}^{\infty} t^{n} \psi_{n}(x) \psi_{n}(y) .
$$

Можно показать, что интегральный оператор $K_{t}: \mathcal{H}_{\mu}^{(1)} \mapsto \mathcal{H}_{\mu}^{(2)}$,

$$
\left(K_{t} f\right)(y)=\int_{-\infty}^{\infty} f(x) \mathfrak{K}_{\mu}(x, y ; t) \mu(d x),
$$

является унитарным оператором при $|t|=1$.

ОПРЕДЕЛЕНИЕ 2.2. Унитарные операторы $F^{ \pm}=K_{\mp i}$, где $i=\sqrt{-1}$, будем называть прямым и обратным обобщенными преобразованиями Фурье, соответствующими системе $\left\{\psi_{n}(x)\right\}_{n=0}^{\infty}$.

Далее введем квантово-механические переменные: операторы "координаты", “импульса" и "гамильтониан".

Будем предполагать, что система $\left\{\psi_{n}(x)\right\}_{n=0}^{\infty}$ ортонормирована и полна в гильбертовом пространстве $\mathcal{H}_{\mu}^{(1)}=L^{2}\left(\mathbb{R}^{1} ; \mu(d x)\right)$. Соотношения (2.3) определяют действие оператора $X_{\mu}$ на базисные векторы $\left\{\psi_{n}(x)\right\}_{n=0}^{\infty}$ в пространстве $\mathcal{H}_{\mu}^{(1)}$. Будем рассматривать этот оператор как оператор “координаты”. Напомним [12], что область определения $D\left(X_{\mu}\right)$ оператора $X_{\mu}$ есть множество всех $f(x) \in \mathcal{H}_{\mu}^{(1)}$ таких, что

$$
\int_{-\infty}^{\infty}|f(x)|^{2}\left(1+x^{2}\right) \mu(d x)<\infty .
$$

Оператор “импульса" $P_{\mu}$ определим следующим образом:

$$
P_{\mu}(t)=K_{\bar{t}} Y_{\mu} K_{t}
$$

где оператор $Y_{\mu}$ - оператор умножения на переменную $y$ в $\mathcal{H}_{\mu}^{(2)}$, определяемый по аналогии с формулами (2.3). При этом мы имеем

$$
D\left(P_{\mu}(t)\right)=K_{\bar{t}} D\left(Y_{\mu}\right)
$$

На плотном в $\mathcal{H}_{\mu}^{(1)}$ множестве $D\left(X_{\mu}\right) \cap D\left(P_{\mu}(t)\right)$ определим оператор

$$
H_{\mu}(t)=\left(X_{\mu}\right)^{2}+\left(P_{\mu}(t)\right)^{2} .
$$

Справедлива следующая теорема. 
Теорема 2.2. Пусть каноническая система $\left\{\psi_{n}(x)\right\}_{n=0}^{\infty}-$ полная ортонормированная система в пространстве $\mathcal{H}_{\mu}^{(1)}$. Эта система является системой собственных функиий самосопряженного оператора $H_{\mu}(t)$ в $\mathcal{H}_{\mu}^{(1)}$, определенного как замыкание оператора (2.12), в том и только в том случае, когда $t=\mp i$. При этом собственные значения оператора $H_{\mu}(\mp i)$ равны

$$
\lambda_{0}=2 b_{0}^{2}, \quad \lambda_{n}=2\left(b_{n-1}^{2}+b_{n}^{2}\right), \quad n \geqslant 1 .
$$

Для доказательства теоремы достаточно непосредственно проверить, что функции $\psi_{n}(x), n=0,1, \ldots$, являются собственными функциями оператора $H_{\mu}(t)$ только в том случае, когда $t=\mp i$. Из условия полноты и ортонормированности системы $\left\{\psi_{n}(x)\right\}_{n=0}^{\infty}$ в пространстве $\mathcal{H}_{\mu}^{(1)}$ следует, что замыкание симметричного оператора $H_{\mu}(\mp i)$ есть самосопряженный оператор (см., например, книгу [12]).

Операторы $P_{\mu}=P_{\mu}^{(1)}=P_{\mu}(-i)$ и $H_{\mu}=H_{\mu}^{(1)}=H_{\mu}(-i)$ в пространстве $\mathcal{H}_{\mu}^{(1)}$ будем называть операторами “импульса" и "гамильтонианом" ортонормированной системы $\left\{\psi_{n}(x)\right\}_{n=0}^{\infty}$. Аналогичные операторы в пространстве $\mathcal{H}_{\mu}^{(2)}$ будем обозначать через $P_{\mu}^{(2)}$ и $H_{\mu}^{(2)}$. Область определения $D\left(H_{\mu}\right)$ оператора $H_{\mu}$ получается из $(2.9)$ и (2.11) замыканием по норме $H_{\mu}$ :

$$
\|f\|_{H_{\mu}}^{2}=\|f\|^{2}+\left\|H_{\mu} f\right\|^{2} .
$$

Следующие две теоремы показывают, что операторы $X_{\mu}, P_{\mu}$ и $H_{\mu}$ обладают теми же свойствами, что и аналогичные им операторы координаты, импульса и гамильтониан для обычного квантово-механического осциллятора.

Теорема 2.3. Действие операторов $X_{\mu}, P_{\mu}, H_{\mu}$ на базисные векторь $\left\{\psi_{n}(x)\right\}_{n=0}^{\infty}$ пространства $\mathcal{H}_{\mu}^{(1)}$ определяется следующими формулами:

$$
\begin{aligned}
& X_{\mu} \psi_{0}(x)=b_{0} \psi_{1}(x), \\
& P_{\mu} \psi_{0}(x)=-i b_{0} \psi_{1}(x), \\
& H_{\mu} \psi_{0}(x)=\lambda_{0} \psi_{0}(x), \\
& X_{\mu} \psi_{n}(x)=b_{n-1} \psi_{n-1}(x)+b_{n} \psi_{n+1}(x), \\
& P_{\mu} \psi_{n}(x)=i\left(b_{n-1} \psi_{n-1}(x)-b_{n} \psi_{n+1}(x)\right), \quad n \geqslant 1, \\
& H_{\mu} \psi_{n}(x)=\lambda_{n} \psi_{n}(x),
\end{aligned}
$$

где собственные значения $\lambda_{n}, n \geqslant 0$, определены формулами (2.13).

Теорема 2.4. В гилъбертовъх пространствах $\mathcal{H}_{\mu}^{(j)}, j=1,2$, справедливъ следующие соотношения для операторов (2.10):

$$
\begin{array}{ll}
P_{\mu}^{(2)} F^{+}=F^{+} X_{\mu}, & Y_{\mu} F^{+}=F^{+} P_{\mu}^{(1)}, \\
P_{\mu}^{(1)} F^{-}=F^{-} Y_{\mu}, & X_{\mu} F^{-}=F^{-} P_{\mu}^{(2)},
\end{array}
$$


и для операторов (2.12):

$$
H_{\mu}^{(2)} F^{+}=F^{+} H_{\mu}^{(1)}, \quad H_{\mu}^{(1)} F^{-}=F^{-} H_{\mu}^{(2)} .
$$

Далеее мы рассматриваем пространство $\mathcal{H}_{\mu}^{(1)}$ как пространство Фока и определим с помощью построенных ранее операторов $X_{\mu}, P_{\mu}$ на плотном в $\mathcal{H}_{\mu}^{(1)}$ множестве $D\left(X_{\mu}\right) \cap D\left(P_{\mu}\right)$ лестничные операторы рождения $a_{\mu}^{+}$и уничтожения $a_{\mu}^{-}$обычным образом:

$$
a_{\mu}^{+}=\frac{1}{\sqrt{2}}\left(X_{\mu}+i P_{\mu}\right), \quad a_{\mu}^{-}=\frac{1}{\sqrt{2}}\left(X_{\mu}-i P_{\mu}\right) .
$$

ЛЕмма 2.1. Операторы (2.24) действуют на векторы базиса пространства $\mathcal{H}_{\mu}^{(1)}$ по формулам $\left(b_{-1}=0\right)$

$$
a_{\mu}^{+} \psi_{n}(x)=\sqrt{2} b_{n} \psi_{n+1}(x), \quad a_{\mu}^{-} \psi_{n}(x)=\sqrt{2} b_{n-1} \psi_{n-1}(x), \quad n \geqslant 0 .
$$

Следующая теорема является следствием теоремы 12.1.3 из книги [12].

Теорема 2.5. Пусть для операторов (2.25), определенных на множестве $D\left(X_{\mu}^{2}\right) \cap D\left(P_{\mu}\right)^{2}$, плотном в гилъбертовом пространстве $\mathcal{H}_{\mu}^{(1)}$, выполняется условие

$$
\left(b_{n+1}^{2}-b_{n-1}^{2}\right)^{2} \leqslant C \min \left\{\left(b_{n+1}^{2}+b_{n}^{2}\right),\left(b_{n}^{2}+b_{n-1}^{2}\right)\right\}, \quad C>0, \quad n \geqslant 0 .
$$

Тогдана

$$
D\left(a_{\mu}^{-*}\right)=D\left(a_{\mu}^{+*}\right)=D\left(H_{\mu}\right)
$$

справедливы следующие равенства:

$$
a_{\mu}^{-*}=\overline{a_{\mu}^{+}}, \quad a_{\mu}^{+*}=\overline{a_{\mu}^{-}},
$$

где *-операчия сопряжения, $\overline{a_{\mu}^{+}} u \overline{a_{\mu}^{-}}-$замъкания операторов $a_{\mu}^{+}$и $a_{\mu}^{-}$.

ОпрЕДЕлЕНиЕ 2.3. Оператором “числа частиц" в пространстве Фока $\mathcal{H}_{\mu}^{(1)}$ с ортонормированным базисом $\left\{\psi_{n}(x)\right\}_{n=0}^{\infty}$ будем называть оператор $N_{\mu}$, который действует стандартным образом на базисные векторы:

$$
N_{\mu} \psi_{n}(x)=n \psi_{n}(x), \quad n \geqslant 0 \text {. }
$$

Симметричный оператор $N_{\mu}$ определен равенствами (2.27) на плотном множестве конечных линейных комбинаций векторов базиса и после замыкания становится самосопряженным оператором, который мы будем по-прежнему обозначать через $N_{\mu}$.

Обозначим через $B\left(N_{\mu}\right)$ оператор-функцию от оператора $N_{\mu}$ в пространстве $\mathcal{H}_{\mu}^{(1)}$, действие которой на векторы базиса $\left\{\psi_{n}(x)\right\}_{n=0}^{\infty}$ описывается следующими формулами:

$$
B\left(N_{\mu}\right) \psi_{n}(x)=b_{n-1}^{2} \psi_{n}(x), \quad n \geqslant 0, \quad b_{-1}=0 .
$$

Для однозначного определения функции $B(z)$, регулярной в правой полуплоскости и непрерывной вплоть до мнимой оси, по ее значениям $B\left(z_{n}\right)=b_{n-1}^{2}, n \geqslant 1$, в точках 
$z_{n}=n, n \geqslant 1$, достаточно выполнения двух условий (см. теорему 3.7.5 в монографии [13]):

$$
\begin{aligned}
& |B(i y)| \leqslant M e^{(\pi-\epsilon)|y|}, \quad-\infty<y<\infty, \quad \epsilon>0, \\
& |B(z)| \leqslant M e^{A|z|}, \quad \operatorname{Re} z>0 .
\end{aligned}
$$

Тогда при достаточно большом $q>0$ функция $B(z)$ может быть представлена в виде

$$
B(z)=\frac{\sin (\pi z)}{\pi z}\left\{\int_{-q}^{\infty} P(x) e^{-z x} d x+e^{q z} \sum_{n=1}^{\infty}(-1)^{n} n b_{n-1}^{2} \frac{e^{-q n}}{z-n}\right\},
$$

где $P(z)$ - аналитическая функция, которую можно представить в полуплоскости $\operatorname{Re} z<a(a-$ достаточно большое по модулю отрицательное число) рядом Дирихле

$$
P(z)=\sum_{n=1}^{\infty}(-1)^{n} n b_{n-1}^{2} e^{z n} .
$$

Следующая теорема позволяет определить алгебру “обобщенного” осциллятора.

Теорема 2.6. Для операторов, определенных формулами (2.24), (2.28) в пространстве Фока $\mathcal{H}_{\mu}^{(1)}$, имеют место соотношения

$$
\left[a_{\mu}^{-}, a_{\mu}^{+}\right]=2\left(B\left(N_{\mu}+I_{\mu}\right)-B\left(N_{\mu}\right)\right), \quad\left[N_{\mu}, a_{\mu}^{ \pm}\right]= \pm a_{\mu}^{ \pm} .
$$

Это утверждение можно получить, используя формулы (2.25), (2.27).

ОПРЕДЕЛЕНИЕ 2.4. Алгебру, порожденную генераторами $a_{\mu}^{ \pm}, N_{\mu}$, которые удовлетворяют перестановочным соотношениям (2.33), мы будем называть в дальнейшем алгеброй обобщенного осциллятора, соответствующего системе полиномов $\left\{\psi_{n}(x)\right\}_{n=0}^{\infty}$, и обозначать через $\mathcal{A}_{\mu}$.

ТЕОРема 2.7. Центр алгебры $\mathcal{A}_{\mu}$ порождается элементом

$$
C=2 B\left(N_{\mu}\right)-a_{\mu}^{+} a_{\mu}^{-}
$$

Доказательство дается непосредственными вычислениями.

Так как определенная выше алгебра обобщенного осциллятора $\mathcal{A}_{\mu}$ имеет нетривиальный центр (2.34), то для нее существует множество неэквивалентных неприводимых представлений в отличие от алгебры Гейзенберга, где в силу теорем единственности Релиха-Диксьмье (см., например, книгу [14]) имеется (с точностью до унитарной эквивалентности) только фоковское представление. Для алгебры деформированного осциллятора такие не эквивалентные фоковским (сингулярные) представления были описаны в работах [15], [16]. Мы будем рассматривать далее подалгебру алгебры $\mathcal{A}_{\mu}$, определяемую дополнительным соотношением $C=0$. При этом из формул $(2.33),(2.34)$ следует, что

$$
\begin{aligned}
& a_{\mu}^{+} a_{\mu}^{-}=2 B\left(N_{\mu}\right), \\
& a_{\mu}^{-} a_{\mu}^{+}=2 B\left(N_{\mu}+I\right) .
\end{aligned}
$$


Поскольку эта подалгебра имеет (с точностью до унитарной эквивалентности) только фоковское представление, мы будем называть ее обобщенной алгеброй Гейзенберга. В данной работе мы имеем дело именно с такой ситуацией. В работах [17]-[20] в рамках предложенного подхода были получены обобщенные осцилляторы для всех классических полиномов непрерывного и дискретного аргумента (полиномы Лагерра, Чебышева, Лежандра, Гегенбауэра, Мейкснера, Шарлье и Кравчука), а также для "деформированных" полиномов Эрмита.

\section{3. НЕСИММЕТРИЧНАЯ СХЕМА}

Пусть положительная борелевская мера $\mu$ на вещественной оси имеет все конечные моменты, но условие симметричности (2.2) не выполняется.

По заданной последовательности моментов $\left\{\mu_{n}\right\}_{n=0}^{\infty}$ теперь будем искать две вещественные последовательности $\left\{b_{n}\right\}_{n=0}^{\infty},\left\{a_{n}\right\}_{n=0}^{\infty}$ как решения следующей системы уравнений:

$$
A_{k, n}=b_{n} A_{k-1, n+1}+a_{n} A_{k-1, n}+b_{n-1} A_{k-1, n-1}, \quad n \geqslant 0, \quad k \geqslant 1, \quad b_{-1}=0,
$$

которые также удовлетворяют условиям

$$
A_{0,0}=1, \quad A_{k, 0}=\mu_{k}, \quad A_{0, n}=0, \quad k, n \geqslant 1 .
$$

Можно показать, что верна следующая лемма.

ЛЕмма 3.1. Система уравнений (3.1) относительно переменных

$$
\left(a_{n}, b_{n}, A_{k, n}\right), \quad n \geqslant 0, \quad k \geqslant 1,
$$

имеет единственное решение, удовлетворяющее условиям (3.2).

По данным последовательностям $\left\{a_{n}\right\}_{n=0}^{\infty},\left\{b_{n}\right\}_{n=0}^{\infty}$ определим каноническую систему полиномов с помощью рекуррентных соотношений

$$
x \psi_{n}(x)=b_{n} \psi_{n+1}(x)+a_{n} \psi_{n}(x)+b_{n-1} \psi_{n-1}(x), \quad n \geqslant 0, \quad b_{-1}=0,
$$

где

$$
\psi_{0}(x)=1
$$

Аналогом теоремы 2.1 для несимметричного случая является следующая теорема.

ТЕОрема 3.1. Пусть $\left\{\psi_{n}(x)\right\}_{n=0}^{\infty}-$ система полиномов, определяемая с помощъю рекуррентных соотношений (3.3), (3.4), и пусть $\mu$ - вероятностная мера на вещественной оси $\mathbb{R}^{1}$, имеющая все конечные моменты. Система полиномов $\left\{\psi_{n}(x)\right\}_{n=0}^{\infty}$ ортонормалъна относительно меры $\mu$ тогда и только тогда, когда коэффичиенты $\left\{a_{n}\right\}_{n=0}^{\infty},\left\{b_{n}\right\}_{n=0}^{\infty}$, входящие в рекуррентные соотношения (3.3), являются решением системы (3.1), (3.2), где $\mu_{k}$ определяются равенствами (2.1). 
При этом система полиномов $\left\{\psi_{n}(x)\right\}_{n=0}^{\infty}$ полна в гилъбертовом пространстве $\mathcal{H}_{\mu}=L^{2}\left(\mathbb{R}^{1} ; \mu(d x)\right)$ только в том случае, когда мера $\mu$ является $N$ - экстремальным решением проблемы моментов, порожденной матрицей $J$, связанной с рекуррентными соотношениями (3.3).

Как и прежде, можно определить оператор импульса $P_{\mu}$, который двойствен оператору координаты $X_{\mu}$ относительно базиса $\left\{\psi_{n}(x)\right\}_{n=0}^{\infty}$ в $\mathcal{H}_{\mu}$, и симметричный гамильтониан $H_{\mu}(t)$, который не будет самосопряженным оператором. Более того, система $\left\{\psi_{n}(x)\right\}_{n=0}^{\infty}$ не является системой собственных функций оператора $H_{\mu}(t)$ ни при каком значении $t$. Тем не менее можно исправить ситуацию, используя новые операторы координаты и импульса. Введем новые операторы координаты $\widetilde{X}_{\mu}$ и импульса $\widetilde{P}_{\mu}$ следующим образом:

$$
\begin{aligned}
\widetilde{X}_{\mu} & =\operatorname{Re}\left(X_{\mu}-P_{\mu}\right), \\
\widetilde{P}_{\mu} & =(-i) \operatorname{Im}\left(X_{\mu}-P_{\mu}\right) .
\end{aligned}
$$

Если мы совершим замены $X_{\mu} \mapsto \widetilde{X}_{\mu}$ и $P_{\mu} \mapsto \widetilde{P}_{\mu}$, то формулы (2.15)-(2.19) остаются справедливыми для операторов $\widetilde{X}_{\mu}$ и $\widetilde{P}_{\mu}$.

Лемма 3.2. Пусть операторь $\tilde{X}_{\mu}$ и $\widetilde{P}_{\mu}$ определенъ с помощъю формул (3.5), (3.6). Тогда имеет место формула (2.10) $(t=i)$ :

$$
\widetilde{P}_{\mu}=K_{\mu}^{*} \widetilde{Y_{\mu}} K_{\mu}
$$

Определим оператор энергии соотношением

$$
\widetilde{H_{\mu}}=\widetilde{X}_{\mu}^{2}+\widetilde{P}_{\mu}^{2}
$$

Аналогом теоремы 2.2 является следующая теорема.

ТЕорема 3.2. Формула (3.8) определяет самосопряженный оператор $\widetilde{H_{\mu}}$ в гилъбертовом пространстве $\mathcal{H}_{\mu}$ с ортонормированным базисом $\left\{\psi_{n}(x)\right\}_{n=0}^{\infty}$. Более того, система $\left\{\psi_{n}(x)\right\}_{n=0}^{\infty}$ является системой собственных функиий оператора $\widetilde{H_{\mu}}$, и собственные значения этого оператора равны

$$
\lambda_{0}=2 b_{0}^{2}, \quad \lambda_{n}=2\left(b_{n-1}^{2}+b_{n}^{2}\right), \quad n \geqslant 1 .
$$

Определим лестничные операторы

$$
\widetilde{a_{\mu}^{+}}=\frac{1}{\sqrt{2}}\left(\widetilde{X}_{\mu}+i \widetilde{P}_{\mu}\right), \quad \widetilde{a_{\mu}^{-}}=\frac{1}{\sqrt{2}}\left(\widetilde{X}_{\mu}-i \widetilde{P}_{\mu}\right) .
$$

Формулы (2.25) остаются справедливыми при замене $a_{\mu}^{ \pm} \mapsto \widetilde{a_{\mu}^{ \pm}}$. Более того, теорема 2.6 также верна.

ЗАмечАниЕ. Отметим, что в общем случае мы также построили некоторую систему, подобную осциллятору. Однако в этом случае оператор "координаты" не является оператором умножения на независимую переменную. 


\section{4. КОГЕРЕНТНЫЕ СОСТОЯНИЯ ДЛЯ ОБОБЩЕННОГО ОСЦИЛЛЯТОРА}

Понятие когерентных состояний занимает одно из центральных мест в современной квантовой физике. Они играют важную роль в квантовой оптике [4] и в квантовой теории поля при исследовании инфракрасных расходимостей в квантовой электродинамике [21]. Введенные для квантово-механического осциллятора [22] (группа Гейзенберга) когерентные состояния определены в настоящее время для широкого класса квантовых физических систем, а также для систем, связанных с другими группами (в том числе супергруппами и квантовыми группами).

Можно выделить несколько основных подходов к определению когерентных состояний.

А. Когерентные состояния определяются как собственные состояния оператора уничтожения $a: a|z\rangle=z|z\rangle, z \in \mathbb{C}$. Такие когерентные состояния часто называют когерентными состояниями типа Барута-Жирарделло, так как в работе этих авторов [23] это определение было перенесено на случай некомпактных групп.

Б. Когерентные состояния рассматриваются как результат действия унитарного оператора сдвига

$$
D(z)=e^{z a^{+}-\bar{z} a}
$$

(здесь $a^{+}, a$ - операторы рождения и уничтожения) на вакуумный вектор $|0\rangle$ пространства Фока: $|z\rangle=D(z)|0\rangle$. Такие когерентные состояния чаще всего называют когерентными состояниями типа Переломова, активно изучавшего их (см. монографию [24]).

В. Когерентные состояния определяются как состояния, минимизирующие соотношение неопределенностей Гейзенберга (или Шредингера-Робертсона).

Г. Когерентные состояния рассматриваются как состояния, удовлетворяющие некоторым естественным условиям:

1) нормируемости, т.е. $\langle z \mid z\rangle=1$;

2) непрерывности по индексу $z$, т.е. $\||z\rangle-\left|z^{\prime}\right\rangle \| \rightarrow 0$, если $\left|z-z^{\prime}\right| \rightarrow 0$;

3) (сверх)полноте, т.е. существованию такой положительной весовой функции $\widehat{W}\left(\left|z^{2}\right|\right)>0$, для которой имеет место следующее равенство (разложение единицы):

$$
\iint_{\mathbb{C}}|z\rangle\langle z| \widehat{W}\left(|z|^{2}\right) d^{2} z=\mathbf{1}
$$

где $d^{2} z=d(\operatorname{Re} z) d(\operatorname{Im} z)$. Такие состояния называют когерентными состояниями типа Клаудера-Газо [25].

Известно, что в случае обычного квантово-механического осциллятора все эти определения порождают одно и то же семейство когерентных состояний, т.е. они 
эквивалентны, однако в общем случае это не так. Конструкция обобщенного осциллятора позволяет осуществить построение когерентных состояний любым из указанных выше способов и выяснить все возможные связи между различными определениями. В работах [17]-[20] были получены явные формулы, выражающие когерентные состояния различных типов через известные специальные функции математической физики, для обобщенных осцилляторов, связанных с вышеупомянутыми полиномами. Основную трудность при построении когерентных состояний представляет доказательство соотношения (пере)полноты соответствующих когерентных состояний, т.е. построение конкретной меры, присутствующей в разложении единицы.

В настоящей работе мы ограничимся рассмотрением только двух типов определений когерентных состояний для обобщенных осцилляторов.

ОПРЕДЕЛЕНИЕ 4.1. Когерентными состояниями типа Барута-Жирарделло называют собственные состояния оператора уничтожения. Для определенного выше обобщенного осциллятора имеем

$$
a^{-}|z\rangle=z|z\rangle, \quad|z\rangle:=\mathcal{N}^{-1 / 2}\left(|z|^{2}\right) \sum_{n=0}^{\infty} \frac{z^{n}}{\left(\sqrt{2} b_{n-1}\right) !}|n\rangle .
$$

Нормирующий множитель $\mathcal{N}\left(|z|^{2}\right)$ равен

$$
\mathcal{N}\left(|z|^{2}\right)=\langle z \mid z\rangle=\sum_{n=0}^{\infty} \frac{|z|^{2 n}}{\left(\sqrt{2} b_{n-1}\right) !} .
$$

ОПРЕДЕЛЕНИЕ 4.2. Когерентные состояния минимизируют соотношение неопределенностей, если неравенство Шредингера-Робертсона

$$
\sigma(X \mid f) \sigma(P \mid f) \geqslant\left|\left\langle f\left|\frac{1}{2}[X, P]\right| f\right\rangle\right|,
$$

для них переходит в равенство. При этом

$$
\begin{aligned}
& \sigma^{2}(X ; f)=\left\langle f\left|X^{2}\right| f\right\rangle-\langle f|X| f\rangle^{2} \\
& \sigma^{2}(P ; f)=\left\langle f\left|P^{2}\right| f\right\rangle-\langle f|P| f\rangle^{2}
\end{aligned}
$$

Справедлива следующая теорема.

ТЕОРема 4.1. Для любого обобщенного осииллятора когерентные состояния типа Барута-Жирарделло минимизируют соотношение неопределенностей. Это означает, что

$$
\sigma(X ; z) \sigma(P ; z)=\left|\left\langle z\left|\frac{1}{2}[X, P]\right| z\right\rangle\right| .
$$

Для доказательства теоремы достаточно вычислить правые части выражений (4.5), (4.6), учитывая (2.19), (2.20), а также (4.2) и (4.3). 
Полученные явные формулы для когерентных состояний позволяют для всех вышеупомянутых систем ортогональных многочленов вычислить некоторые существенные (в квантовой оптике) параметры, например, так называемый параметр Манделя

$$
Q_{\mathrm{M}}(x):=\frac{\left\langle z\left\|N^{2}\right\| z\right\rangle-\langle z\|N\| z\rangle^{2}}{\langle z\|N\| z\rangle}-1
$$

характеризующий отклонение распределения числа возбуждений от пуассоновой статистики. Для когерентных состояний Барута-Жирарделло в работе [26] была получена формула

$$
Q_{\mathrm{M}}(x)=x\left(\frac{\mathcal{N}^{\prime \prime}(x)}{\mathcal{N}^{\prime}(x)}-\frac{\mathcal{N}^{\prime}(x)}{\mathcal{N}(x)}\right), \quad|z|^{2}=x,
$$

которая позволяет определить знак параметра Манделя $Q_{\mathrm{M}}$ (что указывает на характер отклонения статистики возбуждений от пуассоновой). Вычисленный в когерентных состояниях обычного осциллятора, этот параметр принимает нулевое значение, что соответствует пуассоновому распределению. В случае, когда этот параметр принимает положительные значения, говорят о суперпуассоновой статистике возбуждений, а при отрицательных значениях - о субпуассоновой. Можно показать, что для осцилляторов, порождаемых полиномами Шарлье, так же как и для гармонического осциллятора (многочлены Эрмита), параметр Манделя принимает нулевое значение $\left(Q_{\mathrm{M}}=0\right.$; статистика - пуассонова). Для многочленов Лагерра и Мейкснера $Q_{\mathrm{M}}<0$ (субпуассонова статистика). В случае полиномов Гегенбауэра и Кравчука знак параметра Манделя зависит от модуля собственного числа оператора уничтожения соответствующего когерентного состояния. Для деформированных $q$-полиномов Эрмита знак $Q_{\mathrm{M}}$ определяется величиной параметра деформации $q$ : $Q_{\mathrm{M}}<0$ при $0<q<1$, а при $q>1$ значение параметра Манделя положительно, $Q_{\mathrm{M}}>0$.

В следующем разделе мы проиллюстрируем описанный выше метод построения обобщенного осциллятора и когерентных состояний на примере осциллятора, связанного с полиномами Гегенбауэра. Условимся называть такой осциллятор осциллятором Гегенбауэра.

\section{5. КОГЕРЕНТНЫЕ СОСТОЯНИЯ ДЛЯ ОСЦИЛЛЯТОРА ГЕГЕНБАУЭРА}

5.1. Осциллятор Гегенбауэра. Известно, что ультрасферические полиномы

$$
P_{n}^{(\alpha)}(x)=\frac{(\alpha+1)_{n}}{n !}{ }_{2} F_{1}\left(\begin{array}{c}
-n, n+2 \alpha+1 \\
\alpha+1
\end{array} \mid \frac{1}{2}(1-x)\right),
$$


где ${ }_{2} F_{1}$ - гипергеометрическая функция, с точностью до нормировки совпадают с полиномами Гегенбауэра $C_{n}^{\lambda}(x)$ :

$$
\left(\begin{array}{c}
n+2 \lambda-1 \\
n
\end{array}\right) P_{n}^{(\lambda-1 / 2)}(x)=\left(\lambda+\frac{1}{2}\right)_{n} C_{n}^{\lambda}(x) .
$$

Символ Похгаммера [3] $(\beta)_{n}$ определяется как $(\beta)_{0}=1,(\beta)_{n}=\beta(\beta+1) \ldots(\beta+n-1)$, $n \geqslant 1$. Ультрасферические многочлены при $\alpha>-1$ ортогональны:

$$
\int_{-1}^{1} P_{n}^{(\alpha)}(x) P_{m}^{(\alpha)}(x)\left(1-x^{2}\right)^{\alpha} d x=d_{n}^{2} \delta_{n, m}, \quad n, m \geqslant 0,
$$

с нормировочной постоянной

$$
d_{n}^{2}=2^{2 \alpha+1} \frac{(\Gamma(n+\alpha+1))^{2}}{(2 n+2 \alpha+1) n ! \Gamma(n+2 \alpha+1)}, \quad n \geqslant 0 .
$$

Гильбертово пространство $\mathcal{H}$ квадратично-суммируемых функций на промежутке $[-1,1]$ по мере $d_{0}^{-2}\left(1-x^{2}\right)^{\alpha} d x$ имеет ортонормированный базис

$$
\langle x \mid n\rangle=\psi_{n}(x)=\frac{d_{0}}{d_{n}} P_{n}^{(\alpha)}(x), \quad n \geqslant 0 .
$$

Из рекуррентного соотношения для ультрасферических полиномов получаем

$$
x \psi_{n}(x)=b_{n} \psi_{n+1}(x)+b_{n-1} \psi_{n-1}(x), \quad n \geqslant 0 ; \quad \psi_{0}(x)=1,
$$

где

$$
b_{n}=\sqrt{\frac{(n+1)(n+2 \alpha+1)}{(2 n+2 \alpha+1)(2 n+2 \alpha+3)}}, \quad n \geqslant 0 ; \quad b_{-1}=0 .
$$

Мы будем рассматривать гильбертово пространство $\mathcal{H}$ как пространство Фока для системы, подобной осциллятору, которую будем называть осциллятором Гегенбауэра. Оператор координаты $X$ определим как оператор умножения на аргумент в пространстве $\mathcal{H}$. Действие оператора $X$ на элементы базиса в пространстве $\mathcal{H}$ описывается формулой (5.6). Известно (см., например, монографию [11]), что оператор $X$ в гильбертовом пространстве $\mathcal{H}$ существенно самосопряжен. Условимся обозначать замыкание оператора $X$ тем же символом $X$.

Оператор импульса $P$ в $\mathcal{H}$ определим соотношением

$$
P \psi_{n}(x)=i\left(b_{n-1} \psi_{n-1}(x)-b_{n} \psi_{n+1}(x)\right), \quad n \geqslant 0
$$

Далее определим лестничные операторы

$$
a^{+}=\frac{1}{\sqrt{2}}(X+i P), \quad a^{-}=\frac{1}{\sqrt{2}}(X-i P)
$$

которые действуют на элементах базиса $|n\rangle$ в $\mathcal{H}$ согласно формулам

$$
a^{+}|n\rangle=\sqrt{2} b_{n}|n+1\rangle, \quad a^{-}|n\rangle=\sqrt{2} b_{n-1}|n-1\rangle .
$$


Определим далее оператор $N$, нумерующий элементы базиса, и гамильтониан $H$ формулами

$$
\begin{gathered}
N|n\rangle=n|n\rangle, \\
H=X^{2}+P^{2}=a^{(+)} a^{(-)}+a^{(-)} a^{(+)} .
\end{gathered}
$$

Можно проверить, что гамильтониан $H$ - самосопряженный оператор в пространстве $\mathcal{H}$ с собственными значениями

$$
\lambda_{0}=2 b_{0}^{2}, \quad \lambda_{n}=2\left(b_{n-1}^{2}+b_{n}^{2}\right), \quad n \geqslant 1 .
$$

\section{2. Когерентные состояния типа Барута-ЖКирарделло для осциллято-} ра Гегенбауэра. Согласно формулам (5.4), (5.5) для обобщенного осциллятора в нашем случае имеем

$$
\begin{aligned}
\langle x \mid n\rangle= & \frac{\Gamma(\alpha+1)}{\Gamma(2(\alpha+1)) \Gamma(n+\alpha+1)} \times \\
& \quad \times \sqrt{n !(2 n+2 \alpha+1) \Gamma(n+\alpha+1)} P_{n}^{(\alpha)}(x) .
\end{aligned}
$$

Учитывая формулу (5.7), получаем

$$
\left(2 b_{n-1}^{2}\right) !=2^{-n} n ! \frac{(2 \alpha+1)_{n}}{(\alpha+1 / 2)_{n}(\alpha+3 / 2)_{n}}, \quad n \geqslant 1
$$

радиус сходимости ряда в формуле (4.3) равен $1 / \sqrt{2}$ и

$$
\mathcal{N}={ }_{2} F_{1}\left(\begin{array}{c}
\alpha+\frac{1}{2}, \alpha+\left.\frac{3}{2}|2| z\right|^{2} \\
2 \alpha+1
\end{array}\right) \text {. }
$$

Тогда, подставляя выражения (5.16) и (4.3) в формулу (4.2), получаем явный вид когерентных состояний (типа Барута-Жирарделло) для осциллятора Гегенбауэра:

$$
\langle x \mid z\rangle=\frac{1}{(1-x z \sqrt{2})^{(\alpha+3 / 2)}} \frac{{ }_{2} F_{1}\left(\begin{array}{c}
\frac{\alpha}{2}+\frac{3}{4}, \frac{\alpha}{2}+\frac{5}{4}\left|\frac{2 z^{2}\left(x^{2}-1\right)}{(\alpha+1)}\right|(1-x z \sqrt{2})^{2}
\end{array}\right)}{\sqrt{{ }_{2} F_{1}\left(\begin{array}{c}
\left.\alpha+\frac{1}{2}, \alpha+\frac{3}{2}\right)\left.|2| z\right|^{2} \\
2 \alpha+1
\end{array}\right)}} .
$$

Следующая задача состоит в построении меры

$$
d \mu\left(|z|^{2}\right)=W\left(|z|^{2}\right) d^{2} z
$$

в разложении единицы

$$
\iint_{\mathbb{C}_{1 / \sqrt{2}}} W\left(|z|^{2}\right)|z\rangle\langle z| d^{2} z=1
$$


где $d^{2} z=d(\operatorname{Re} z) d(\operatorname{Im} z)$. Известно, что для этого следует решить классическую проблему моментов, имеющую в нашем случае вид

$$
\int_{0}^{1 / 2} t^{n} \widehat{W}(t) d t=\frac{1}{\pi}\left(2 b_{n-1}^{2}\right) !, \quad W(t)=\widehat{W}(t) \mathcal{N} .
$$

Используя формулу (5.15), перепишем равенство (5.20) в виде

$$
\int_{0}^{1 / 2} t^{n} \widehat{W}(t) d t=\frac{1}{\pi} \frac{n !(2 \alpha+1)_{n}}{2^{n}(\alpha+1 / 2)_{n}(\alpha+3 / 2)_{n}}, \quad n \geqslant 0 .
$$

Делая замену переменных $\tau=2 t$ и обозначая

$$
\widetilde{W}(\tau)=\frac{\pi}{2} \frac{\Gamma(2 \alpha+1)}{\Gamma(\alpha+1 / 2) \Gamma(\alpha+3 / 2)} \widehat{W}\left(\frac{1}{2} \tau\right),
$$

перепишем формулу (5.21) в виде

$$
\int_{0}^{1} \tau^{n} \widetilde{W}(\tau) d \tau=\frac{\Gamma(n+1) \Gamma(n+2 \alpha+1)}{\Gamma(n+\alpha+1 / 2) \Gamma(n+\alpha+3 / 2)} .
$$

Для решения указанной проблемы моментов рассмотрим интеграл (7.512.4) из книги [27]:

$$
\int_{0}^{1} x^{\gamma-1}(1-x)^{\rho-1}{ }_{2} F_{1}\left(\begin{array}{c}
\nu, \beta \\
\gamma
\end{array} \mid x\right) d x=\frac{\Gamma(\gamma) \Gamma(\rho) \Gamma(\gamma+\rho-\nu+\beta)}{\Gamma(\gamma+\rho-\nu) \Gamma(\gamma+\rho-\beta)},
$$

где предполагается, что

$$
\operatorname{Re} \gamma>0, \quad \operatorname{Re} \rho>0, \quad \operatorname{Re}(\gamma+\rho-\alpha-\beta)>0
$$

Выбирая следующие значения параметров:

$$
\gamma=1, \quad \rho=n+2 \alpha+1, \quad \nu=\alpha-\frac{1}{2}, \quad \beta=\alpha+\frac{3}{2},
$$

и делая замену переменных $x=1-\tau$ в этом интеграле, при $\alpha>-1 / 2$ получаем

$$
\int_{0}^{1} \tau_{2}^{n+2 \alpha} F_{1}\left(\begin{array}{c}
\alpha-\frac{1}{2}, \alpha+\frac{3}{2} \\
1
\end{array} \mid 1-\tau\right) d \tau=\frac{\Gamma(1) \Gamma(n+2 \alpha+1) \Gamma(n+1)}{\Gamma(n+\alpha+5 / 2) \Gamma(n+\alpha+1 / 2)} .
$$

Поскольку

$$
P_{\frac{1}{2}-\alpha}^{(0,2 \alpha)}(x)={ }_{2} F_{1}\left(\begin{array}{c|c}
\alpha-\frac{1}{2}, \alpha+\frac{3}{2} \mid & \frac{1}{2}(1-x) \\
1
\end{array}\right)
$$

интеграл (5.26) принимает вид

$$
\int_{0}^{1} \tau^{n+2 \alpha} P_{\frac{1}{2}-\alpha}^{(0,2 \alpha)}(1-\tau) d \tau=\frac{\Gamma(n+1) \Gamma(n+2 \alpha+1)}{(n+\alpha+3 / 2) \Gamma(n+\alpha+1 / 2) \Gamma(n+\alpha+3 / 2)} .
$$


Учитывая, что

$$
P_{\nu}^{(\alpha, \beta)}(1)=\frac{\Gamma(\nu+\alpha+1)}{\Gamma(\nu+1) \Gamma(\alpha+1)}, \quad P_{\frac{1}{2}-\alpha}^{(0,2 \alpha)} 1=1
$$

получаем

$$
\lim _{\tau \rightarrow 1^{-}} \tau^{\alpha-1 / 2} P_{1 / 2-\alpha}^{(0,2 \alpha)}(2 \tau-1)=1 .
$$

Определим функцию $q(t)$, положив

$$
\tau^{\alpha-1 / 2} P_{1 / 2-\alpha}^{(0,2 \alpha)}(2 \tau-1)-1=\int_{\tau}^{1} q(t) d t
$$

Дифференцируя это соотношение по $\tau$, находим функцию $q(\tau)$ на отрезке $[0,1]$ :

$$
\begin{aligned}
q(\tau) & =-\left(\tau^{\alpha-1 / 2} P_{1 / 2-\alpha}^{(0,2 \alpha)}(2 \tau-1)\right)_{\tau}^{\prime}= \\
& =\left(\frac{1}{2}-\alpha\right) \tau^{\alpha-3 / 2} P_{1 / 2-\alpha}^{(0,2 \alpha)}(2 \tau-1)-\tau^{\alpha-1 / 2}\left(P_{1 / 2-\alpha}^{(0,2 \alpha)}(2 \tau-1)\right)_{\tau}^{\prime} .
\end{aligned}
$$

Учитывая формулу для производной гипергеометрической функции

$$
\frac{d}{d z}{ }_{2} F_{1}\left(\begin{array}{c}
a, b \\
c
\end{array} \mid z\right)=\frac{a b}{c}{ }_{2} F_{1}\left(\begin{array}{c}
a+1, b+1 \\
c+1
\end{array} \mid z\right)
$$

имеем

$$
P_{1 / 2-\alpha}^{(0,2 \alpha)}(2 \tau-1)_{\tau}^{\prime}=\left(\alpha+\frac{3}{2}\right) P_{-1 / 2-\alpha}^{(1,2 \alpha+1)}(2 \tau-1) .
$$

Подставляя это выражение в (5.32), получаем

$$
q(\tau)=\left(\frac{1}{2}-\alpha\right) \tau^{\alpha-3 / 2} P_{1 / 2-\alpha}^{(0,2 \alpha)}(2 \tau-1)-\left(\alpha+\frac{3}{2}\right) \tau^{\alpha-1 / 2} P_{-\alpha-1 / 2}^{(1,1+2 \alpha)}(2 \tau-1) .
$$

Из формул (5.28) и (5.31) имеем

$$
\begin{gathered}
\int_{0}^{1} \tau^{n+\alpha+1 / 2}\left(\int_{\tau}^{1} q(t) d t\right) d \tau+\int_{0}^{1} \tau^{n+\alpha+1 / 2} d \tau= \\
=\frac{\Gamma(n+1) \Gamma(n+2 \alpha+1)}{(n+\alpha+3 / 2) \Gamma(n+\alpha+1 / 2) \Gamma(n+\alpha+3 / 2)} .
\end{gathered}
$$

После интегрирования по частям первого интеграла в левой части этого соотношения получаем

$$
\int_{0}^{1} \tau^{n+\alpha+3 / 2} q(\tau) d \tau+1=\frac{\Gamma(n+1) \Gamma(n+2 \alpha+1)}{\Gamma(n+\alpha+1 / 2) \Gamma(n+\alpha+3 / 2)} .
$$

Сравнивая эту формулу с формулой (5.23), видим, что

$$
\widetilde{W}(\tau)=\tau^{\alpha+3 / 2} q(\tau)+2 \delta(\tau-1) .
$$


С учетом соотношения

$$
\Gamma(2 \alpha+1)=2^{2 \alpha} \pi^{-1 / 2} \Gamma\left(\alpha+\frac{1}{2}\right) \Gamma(\alpha+1)
$$

при $t=2 \tau$ имеем

$$
\widehat{W}(t)=\frac{\Gamma(\alpha+3 / 2)}{\sqrt{\pi} 2^{2 \alpha-1} \Gamma(\alpha+1)}\left(2^{\alpha+3 / 2} t^{\alpha+3 / 2} q(2 t)+2 \delta(2 t-1)\right)
$$

или, принимая во внимание формулу (5.35), получаем

$$
\begin{aligned}
\widehat{W}(t)= & \frac{\Gamma(\alpha+3 / 2)}{\sqrt{\pi} 2^{2 \alpha-1} \Gamma(\alpha+1)}\left[\left(\frac{1}{2}-\alpha\right) 2^{2 \alpha} t^{2 \alpha} P_{1 / 2-\alpha}^{(0,2 \alpha)}(4 t-1)-\right. \\
& \left.-\left(\alpha+\frac{3}{2}\right) 2^{2 \alpha+1} t^{2 \alpha+1} P_{-1 / 2-\alpha}^{(1,2 \alpha+1)}(4 t-1)+2 \delta(2 t-1)\right] .
\end{aligned}
$$

Из этого следует, что мера $d \mu$ в разложении единицы (5.19) равна

$$
\begin{aligned}
& d \mu\left(|z|^{2}\right)=\frac{2 \Gamma(\alpha+3 / 2)}{\sqrt{\pi} \Gamma(\alpha+1)} \cdot{ }_{2} F_{1}\left(\begin{array}{c}
\left.\alpha+\frac{1}{2}, \alpha+\left.\frac{3}{2}|2| z\right|^{2}\right) \times \\
2 \alpha+1
\end{array}\right) \\
& \quad \times\left\{\left[\left(\frac{1}{2}-\alpha\right)|z|^{4 \alpha} P_{1 / 2-\alpha}^{(0,2 \alpha)}\left(4|z|^{2}-1\right)-\right.\right. \\
& \left.\left.\quad-2\left(\frac{3}{2}+\alpha\right)|z|^{4 \alpha+2} P_{-1 / 2-\alpha}^{(1,2 \alpha+1)}\left(4|z|^{2}-1\right)\right]+2 \delta\left(2|z|^{2}-1\right)\right\} d^{2} z
\end{aligned}
$$

Вычисляя перекрытие когерентных состояний, получаем

$$
\left\langle z_{1} \mid z_{2}\right\rangle=\frac{{ }_{2} F_{1}\left(\begin{array}{c}
\left.\alpha+\frac{1}{2}, \alpha+\frac{3}{2} \mid 2 \bar{z}_{1} z_{2}\right) \\
2 \alpha+1
\end{array} \mid\right.}{\sqrt{{ }_{2} F_{1}\left(\left.\begin{array}{c}
\alpha+\frac{1}{2}, \alpha+\frac{3}{2} \\
2 \alpha+1
\end{array}|2| z_{1}\right|^{2}\right){ }_{2} F_{1}\left(\begin{array}{c}
\alpha+\frac{1}{2}, \alpha+\frac{3}{2} \mid \\
2 \alpha+1
\end{array}\left|z_{2}\right|^{2}\right)}}
$$

Благодарности. Автор благодарен В. М. Бабичу, П. П. Кулишу и С. Ю. Славянову за полезные дискуссии, а также Е. В. Дамаскинскому за плодотворное сотрудничество.

\section{Список литературы}

[1] Г. Сеге, Ортогональные многочлены, Физматгиз, М., 1962.

[2] С. Ю. Славянов, В. Лай, Специалъные функиии. Единая теория, основанная на анализе особенностей, Невский диалект, СПб., 2002.

[3] R. Koekoek, R. F. Swarttouw, The Askey-scheme of hypergeometric orthogonal polynomials and its q-analogue, Report 94-05, Delft Univ. Technology, 1994.

[4] V. V. Dodonov, J. Opt. B, 4:1 (2002), R1.

[5] Н. М. Атакишиев, С. К. Суслов, ТМФ, 85:1 (1990), 64.

[6] M. Arik, D. D. Coon, J. Math. Phys., 17 (1976), 524. 
[7] L. C. Biedenharn, J. Phys. A, 22 (1989), L873.

[8] A. J. Macfarlane, J. Phys. A, 22 (1989), 4581.

[9] Е. В. Дамаскинский, П. П. Кулиш, Зап. научн. сем. ЛОМИ, 189 (1991), 37.

[10] V. V. Borzov, Integral Transform. Spec. Funct., 12:2 (2001), 115.

[11] Н. И. Ахиезер, Классическая проблема моментов и некоторые вопросы анализа, связанные с нею, Физматгиз, М., 1961.

[12] М.Ш. Бирман, М.З. Соломяк, Спектральная теория самосопряженных операторов в гилъбертовом пространстве, Изд-во ЛГУ, Л., 1980.

[13] М. А. Евграфов, Асимптотические оценки и целье функиии, Наука, М., 1979.

[14] C. R. Putnam, Commutation properties of Hilbert space operators, Ergeb. Math. Grenzgeb., 36, Springer, Berlin-New York, 1967.

[15] П. П. Кулиш, ТМФ, 86:1 (1991), 157.

[16] В. В. Борзов, Е. В. Дамаскинский, С. Б. Егоров, Зап. научн. сем. ПОМИ, 245 (1997), 80.

[17] В. В. Борзов, Е. В. Дамаскинский, Зап. научн. сем. ПОМИ, 308 (2004), 48.

[18] В.В.Борзов, Е. В. Дамаскинский, Зап. научн. сем. ПОМИ, 317 (2004), 66.

[19] В.В. Борзов, Е.В. Дамаскинский, "Charlier polynomials and Charlier oscillator as discrete realization of the harmonic oscillator", Проблемы математического анализа, Вып. 30, ред. Н. Н. Уральцева, Тамара Рожковская, Новосибирск, 2005, 3.

[20] В. В. Борзов, Е. В. Дамаскинский, Зап. научн. сем. ПОмИ, 335 (2006), 75.

[21] П. П. Кулиш, Л. Д. Фаддеев, ТМФ, 4:2 (1970), 153.

[22] E. Schrödinger, Naturwissenshaften, 14 (1926), 644.

[23] A. O. Barut, L. Girardello, Commun. Math. Phys., 21:1 (1971), 41.

[24] А.М. Переломов, Обобщенные когерентные состояния и их применения., Наука, М., 1987.

[25] J. P. Gazeau, J. R. Klauder, J. Phys. A, 32:1 (1999), 123.

[26] В.В. Борзов, Е.В. Дамаскинский, Вычисление параметра Манделя для обобщенных когерентных состояний деформированных осиилляторов, связанных с ортогональными полиномами, Препринт ПОМИ РАН № 21, 2005.

[27] И.С. Градштейн, И.М. Рыжик, Таблицы интегралов, сумм, рядов и произведений, ГИФМЛ, М., 1963. 